\title{
Preparation of Core-Corona Type Microgels of Polyacrylamide
}

\author{
Kong Soo KIM, Suk Hyeong CHO,* and Young Jun KIM** \\ Department of Industrial and Engineering Chemistry, \\ Faculty of Engineering, Chungbuk National University, \\ CheongJu 360-763, Korea \\ * Department of Chemical Engineerning, Chungbuk National University, \\ CheongJu 360-763, Korea \\ ** Department of Industrial and Engineering Chemistry, Hyejeon Junior College, \\ Hong Seong-Eup 350-800, Korea
}

(Received February 15, 1993)

\begin{abstract}
Precipitation polymerization of acrylamide (AAm) and acryloyl terminated PEG macromonomer (AtPEGM) was carried out in selective solvents such as alcohol. Fine particles of microgels were obtained according to the mole ratio of AtPEGM and AAm. The particle size of microgels decreased with increasing concentration of AtPEGM and increased with that of AAm. It was found that microgels were stabilized by steric stabilization of AtPEGM and have the morphology of core-corona type with the size distribution of submicron.

KEY WORDS Precipitation Polymerization / Macromonomer / Microsphere

/ Core-Corona type / Microgel / Hydrogel /
\end{abstract}

Polymer microgels are important as toughening agents for surface coatings and adhesives. Recently, in addition to ordinary uses, some special needs for the microgels have increased in fields such as biology, medicine, phamacology, etc. ${ }^{1-5}$

In general, polymer microgels are usually obtained by emulsion polymerization, ${ }^{6,7}$ seed polymerization, ${ }^{8}$ precipitation polymerization, ${ }^{9,10}$ electrocapillary emulsification method, ${ }^{11}$ etc. Especially, in precipitation polymerization, polymers precipitate in the cause of polymerization and usually form coagulums or aggregates instead of fine particles. However, in the precipitation polymerization of some $\mathrm{N}$-substituted acrylamides and acrylamide with some comonomer, a few exceptional cases were found by Penlton ${ }^{9}$ and Kawaguchi ${ }^{12}$ in which monodisperse hydrogel microspheres were obtained.

In this study, acryloyl terminated PEG macromonomer (AtPEGM) was synthesized and precipitation polymerization of acylamide with AtPEGM was carried out in selective solvents. The roles of PEG macromonomer were studied and the mechanism for formation of polyacrylamide microgels is discussed.

\section{EXPERIMENTAL}

\section{Materials}

Acrylamide was recrystallized from aceton. $N N^{\prime}$-methylene-bis-acrylamide (MBAm) was recrystallized from methanol and acryloyl chloride (Tokyo Kasei Co.) was used as received. Polyethyleneglycol (PEG, $M_{w}$ 750) was dried under a reduced pressure at $50^{\circ} \mathrm{C}$. Benzoyl peroxide (BPO) was used without further furification. Benzene, ethanol, methanol, dimethylformamide (DMF), and dimethyl acetamide (DMAc) were distilled before use.

\section{Synthesis of AtPEGM}

PEG $(0.02 \mathrm{~mol}, 15 \mathrm{~g})$ and triethylamine $(0.02 \mathrm{~mol}, 2.2 \mathrm{ml})$ in $50 \mathrm{ml}$ of benzene were placed into $100 \mathrm{ml}$ three necked flask. Acryloyl 
chloride $(0.021 \mathrm{~mol}, 1.84 \mathrm{ml})$ in $20 \mathrm{ml}$ benzene was slowly under nitrogen atmosphere. The reaction mixture was stirred at $0^{\circ} \mathrm{C} \sim 10^{\circ} \mathrm{C}$ for $12 \mathrm{~h}$ and into an exess of diethyl ether. AtPEGM was collected on a glass filter and washed with diethyl ether, and dried at room temperature under vacuum.

${ }^{1} \mathrm{H}$ NMR ( $\delta$ in $\mathrm{CDCl}_{3}$ ): 6.26 (qt, $1 \mathrm{H}$, $=\mathrm{CH}-), 6.41\left(\mathrm{qt}, 1 \mathrm{H}\right.$, cis $\left.\mathrm{CH}_{2}=\right), 5.84(\mathrm{qt}, 1 \mathrm{H}$, trans $\mathrm{CH}_{2}=$ ), 3.6 (qt, $1 \mathrm{H},-\mathrm{OCH}_{2} \mathrm{CH}_{2}-$ ) ppm (TMS).

\section{Polymerization}

Given amounts of AtPEGM, AAm, MBAm, and BPO were dissolved in $50 \mathrm{ml}$ of ethanol. Polymerization was carried out by adding $4.0 \times 10^{-3} \mathrm{moll}^{-1}$ of BPO initiator at $60^{\circ} \mathrm{C}$ for $6 \mathrm{~h}$ under nitrogen atmosphere. The product was collected by centrifugation, washed with methanol 3 times and dried at room temperature under vacuum.

\section{Measurements}

The size distribution of polymer particles were measured using electron micrographs (Hitachi S-530) and Malvern Autosizer. The shape of polymer particles was observed on scanning electron micrographs (SEM) and transmission electron micrographs (TEM) (Hitachi H-600). Intrinsic viscosity of polyacrylamide microgels in water was measured by Ostwald viscometer.

\section{RESULTS AND DISCUSSION}

AtPEGM was obtained by reaction of PEG with acryloyl chloride. The reaction was performed under mild conditions such as $0^{\circ} \mathrm{C} \sim 10^{\circ} \mathrm{C}$ for $12 \mathrm{~h}$ to avoid the polymerization in the course of macromonomer synthesis. The resulting AtPEGM was soluble in water, methanol, ethanol, DMF, DMAc, and benzene but insoluble in ether and THF.

The absorbtion band by vinyl group at $1620 \mathrm{~cm}^{-1}$ and carbonyl band $1720 \mathrm{~cm}^{-1}$ appeared and that by hydroxyl group at
Table I. Precipitation polymerization of polyacrylamide in selective solvents ${ }^{a}$

\begin{tabular}{cccccc}
\hline \multirow{2}{*}{$\begin{array}{c}\text { Run } \\
\text { No. }\end{array}$} & \multicolumn{2}{c}{ Feed $/ \times 10^{-2} \mathrm{mol1}^{-1}$} & & Yields \\
\cline { 2 - 5 } & AtPEGM & AAm & MBAm & & $\%$ \\
\hline 1 & 1.0 & 18.2 & 0.8 & DMF & $-^{\mathrm{b}}$ \\
2 & 1.0 & 18.2 & 0.8 & DMAc & $\overline{-}^{\mathrm{b}}$ \\
3 & 1.0 & 18.2 & 0.8 & Ethanol & 41.2
\end{tabular}

${ }^{\mathrm{a}}[\mathrm{BPO}]=4.0 \times 10^{-3} \mathrm{moll}^{-1}$.

b Not collected.

Table II. Precipitation polymerization of Polyacrylamide in ethanol at $60^{\circ} \mathrm{C}^{\mathrm{a}}$

\begin{tabular}{rccccc}
\hline \multirow{2}{*}{$\begin{array}{r}\text { Run } \\
\text { No. }\end{array}$} & \multicolumn{2}{c}{ Feed $/ \times 10^{-2} \mathrm{moll}^{-1}$} & & Yields \\
\cline { 2 - 3 } \cline { 5 - 6 } & AtPEGM & AAm & MBAm & & $\%$ \\
\hline 4 & 6.0 & 13.2 & 0.8 & & $-^{\mathrm{b}}$ \\
5 & 4.0 & 15.2 & 0.8 & & $-^{\mathrm{b}}$ \\
6 & 1.4 & 17.8 & 0.8 & & 0.8 \\
7 & 1.0 & 18.2 & 0.8 & & 41.2 \\
8 & 0.4 & 18.8 & 0.8 & & 80.8 \\
9 & 0.2 & 19.0 & 0.8 & & $95.4^{\mathrm{c}}$ \\
10 & 1.0 & 25.0 & 4.0 & & 65.0 \\
11 & 1.0 & 35.0 & 4.0 & & 83.4 \\
12 & 1.0 & 45.0 & 4.0 & & 95.2 \\
\hline
\end{tabular}

a $[\mathrm{BPO}]=4.0 \times 10^{-3} \mathrm{moll}^{-1}$.

${ }^{\mathrm{b}}$ Not collected.

c Aggregated.

$3400 \mathrm{~cm}^{-1}$ was not appeared. From the IR spectrum, it was confirmed that AtPEGM was synthesized.

The precipitation polymerization of AAm with AtPEGM was carried out in selective solvents such as DMF, DMAc, and ethanol. These solvents were good solvents for PEG macromonomer but not polyacrylamide. As shown in Table I, the products of precipitation polymerizations were not collected in DMF and DMAc but only obtained in ethanol. It was found that the selection of solvent is important, and ethanol was a suitable solvent in this system.

The results of polymerization on various concentration of AtPEGM are shown in Table II. The microgels were not collected when the 

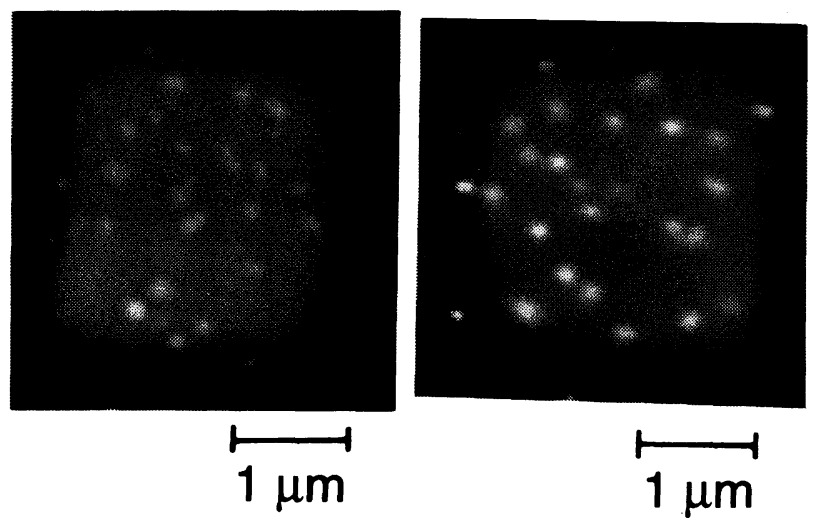

Run 6
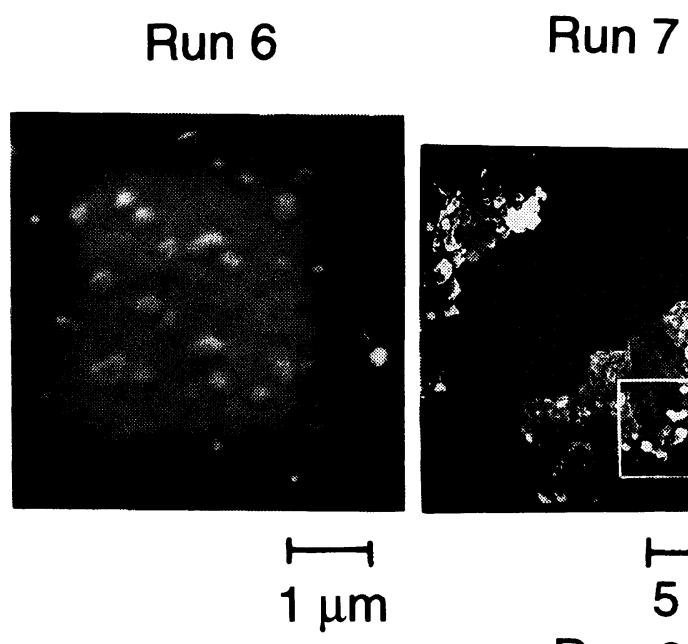

Run 8

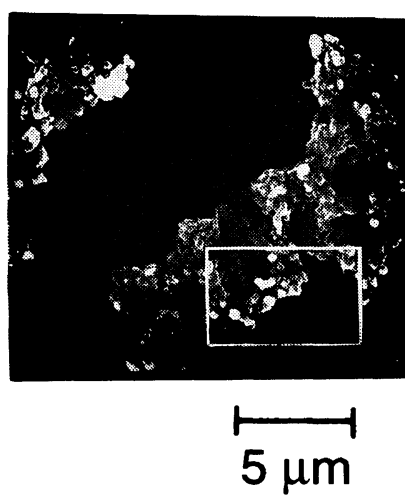

Run 9

Figure 1. Scanning electron micrographs of polyacrylamide microgels.

concentration of AtPEGM was more than $5 \mathrm{~mol} \%$. The microgels were swollen and dispersed in the ethanol and the microgels seems soluble with increasing with AtPEGM. The microgels were aggregated when the concentration of AtPEGM. was less than $1 \mathrm{~mol} \%$. It was found that the microgels are stabilized by steric stabilization of AtPEGM.

The electron micrographs of the microgels are shown in Figures 1 and 2. As showed in Figure 1, polymer particles were distributed homogeneously with the size of $0.2-0.5 \mu \mathrm{m}$ diameter. However, it was shown that polymer products obtained from Run No. 9 aggregated.

In order to stain PEG sequences of the microgel, the microgels were dispersed in water, and a drop of $\mathrm{OsO}_{4}(10 \mathrm{wt} \% \mathrm{aq})$ was added. A drop of the stained microgel solution was placed on a copper grid coated with carbon film, and then dried as gradually at $25^{\circ} \mathrm{C}$. As shown in Figure 2, it was confirmed that microgels have the core-corona type morphology. The core areas represent polyacrylamide regions, and the corona areas are the PEG macromonomer regions.

From the results of electron micrographs of microgels, it is considered that microgels are formed by the mechanism shown in Figure 3. First, the polymer micelle is formed from the copolymerization of AtPEGM and AAm in a 

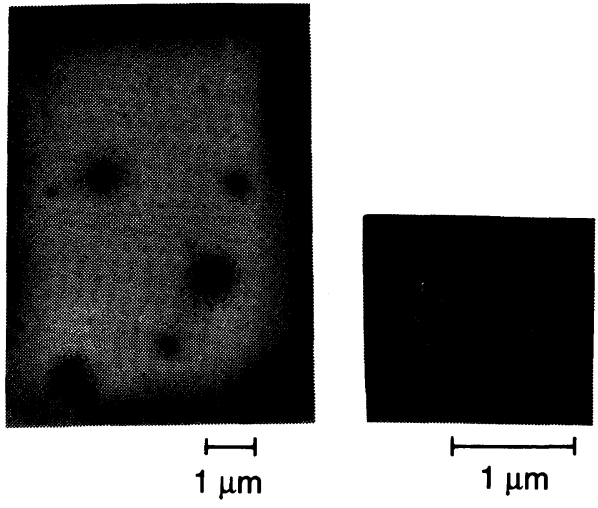

Figure 2. Transmission electron micrographs of polyacrylamide microgels.

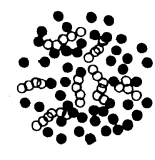

(A)

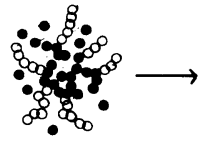

(B)

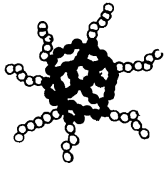

(C)

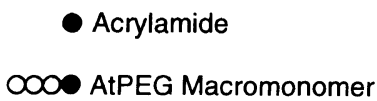

Figure 3. Mechanism for the formation of polyacrylamide microgels in ethanol.

selective solvents. Second, the core area of the polymer micelle is crosslinked by MBAm. This crosslinked polymer micelle formed microgels of the core-corona type.

We investigated the influence of mol ratio of AtPEGM, AAm, and MBAm on the size distribution of the microgels as shown the Figure 4. The size of microgels decreased with increasing concentration of AtPEGM. It was excepted that AtPEGM acts as a stabilizer to form stable microgels of small particle size.

As shown in the Figure 5 microgel size increased with the concentration of the AAm. The gel particles may contains large amount of monomer if the large amount of monomer is charged. The equilibrated amount of monomer absorbed in gel particles is poly-

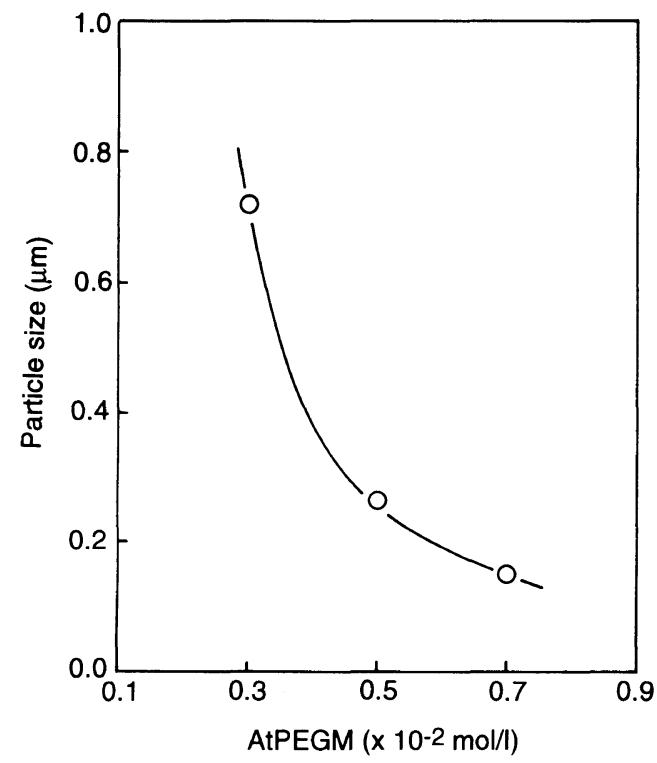

Figure 4. Influence of AtPEGM concentration of polyacrylamide microgel size ([AAm], $18.2 \times 10^{-2} \mathrm{moll}^{-1}$; [MBAm], $\left.0.8 \times 10^{-2} \mathrm{moll}^{-1}\right)$.

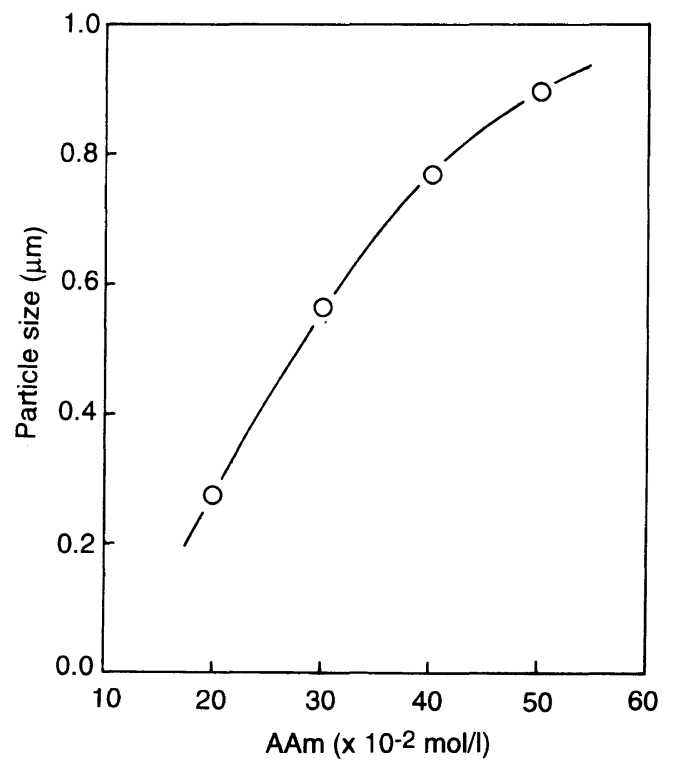

Figure 5. Influence of AAm concentration on polyacrylamide microgel size ([AtPEGM], $1.0 \times 10^{-2} \mathrm{moll}^{-1}$; [MBAm], $\left.0.8 \times 10^{-2} \mathrm{moll}^{-1}\right)$.

merized. It is considered that the particle size is determined by the amount of monomer.

The influence of the amount of MBAm is 


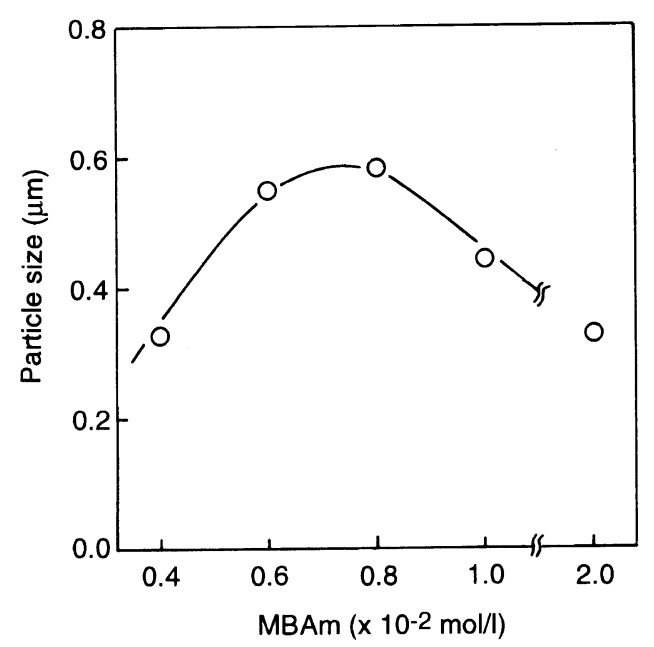

Figure 6. Influence of MBAm concentration on polyacrylamide microgel size ([AtPEGM], $1.0 \times 10^{-2} \mathrm{moll}^{-1}$; [AAm], $18.2 \times 10^{-2} \mathrm{moll}^{-1}$ ).

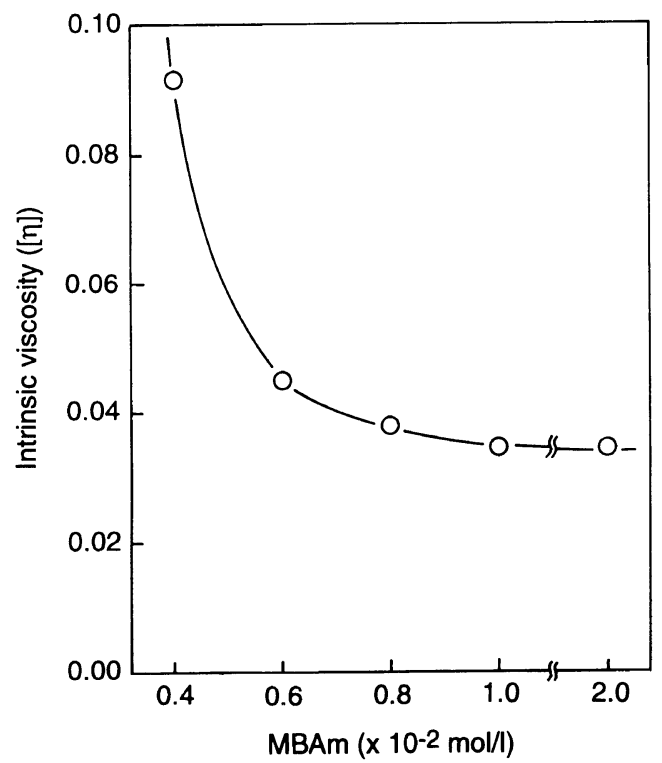

Figure 7. Intrinsic viscosity of polyacrylamide microgels versus $\mathrm{MBAm}$ concentration $\left(\mathrm{H}_{2} \mathrm{O}, 30^{\circ} \mathrm{C}\right)$.

shown in Figure 6. Microgel size increased up to a MBAm concentration of $0.8 \times 10^{-2}$ moll ${ }^{-1}(4 \mathrm{~mol} \%)$. However, it decreased at higher concentration. This MBAm has to conflicting effects ${ }^{13}$ on the size of polymer particles in precipitation polymerization. One is a sweiling effect which increases swelling of the microgels copolymerized with MBAm. The other is a tighening effect which makes dense particles by higher crosslinking. In this system, it was noted that the former effect appeares at a low concentration of MBAm and the latter, at a higher concentration.

The average diameter of the microgels could be controlled from $0.1 \mu \mathrm{m}$ to $1.0 \mu \mathrm{m}$ varying the compositions of AAm, AtPEGM, and MBAm in selective solvents. As shown in Figure 7, the intrinsic viscosity of the microgels decreased with increasing concentration of MBAm. This was due to increase in the branching points according to crosslinking degree. The intrinsic viscosity of the microgels was very low.

\section{REFERENCES}

1. T. Kawaguchi, A. Tsuganz, A. Higashide, H. Endoh, T. Hasekawa, H. Kanno, T. Seki, K. Juni, S. Hukushima, and M. Makano, J. Pham. Sci., 81, 508 (1992)

2. C. X. Song, H. F. Sun, and X. D. Feng, Polym. J., 19, 485 (1987).

3. P. Edman and I. Sjohlm, J. Pham. Sci., 72, 654 (1983).

4. H. Abe, Y. Yamamoto, and Y. Doi, Kobunshi Ronbunshu, 49, 61 (1992).

5. S. Kame, M. Okubo, T. Matsuda, and . Matanoto, Colloid Polym. Sci., 264, 743 (1986); F. Sundardi, A. Zubir, Y. Sabarinah, and W. Sofiarti, J. Macromol. Sci. Chem., A24, 1369 (1987).

6. F. Candau and Y. S. Leong, J. Polym. Sci., A, 23, 193 (1985)

7. W. Bade and K. H. Reichert, Eur. Polym. J., 20, 505 (1984).

8. M. Okubo, Kobumshi, 40, 704 (1991).

9. R. F. Penlton and P. Chibante, Colloids and Surface, 20, 247 (1986).

10. E. F. Tai, J. Polym. Sci., A, 24, 567 (1986).

11. S. Hayashi, A. Komatsu, and T. Hirai, J. Polym. Sci., $A, 27,157$ (1989).

12. H. Kawaguchi, Y. Yamada, S. Kataoka, Y. Morita, and Y. Ohtsuka, Polym. J., 23, 955 (1991).

13. H. Kawaguchi, M. Kawahara, N. Yaguchi, F. Hoshino, and Y. Ohtsuka, Polym. J., 20, 903 (1988). 